\title{
LANDSAT 9 THERMAL INFRARED SENSOR 2 CHARACTERIZATION PLAN OVERVIEW
}

\author{
Joel McCorkel ${ }^{1}$, Matthew Montanaro' ${ }^{2}$,Boryana Efremova ${ }^{3}$, Aaron Pearlman ${ }^{3}$, Brian Wenny ${ }^{4}$, \\ Allen Lunsford ${ }^{5}$, Amy Simon ${ }^{1}$, Jason Hair ${ }^{1}$, Dennis Reuter ${ }^{1}$ \\ 'NASA Goddard Space Flight Center, Greenbelt, MD 20771, USA \\ ${ }^{2}$ Rochester Institute of Technology, Rochester, NY 14623, USA \\ ${ }^{3}$ GeoThinkTank LLC, Washington, DC 20009, USA \\ ${ }^{4}$ Science Systems and Applications, Inc., Greenbelt, MD 20771, USA \\ ${ }^{5}$ Catholic University of America, Washington, DC 20064, USA
}

\begin{abstract}
Landsat 9 will continue the Landsat data record into its fifth decade with a near-copy build of Landsat 8 with launch scheduled for December 2020. The two instruments on Landsat 9 are Thermal Infrared Sensor-2 (TIRS-2) and Operational Land Imager-2 (OLI-2). TIRS-2 is a twochannel pushbroom imager with a 15-degree field of view that will have a 16-day measurement cadence from its nominal $705-\mathrm{km}$ orbit altitude. Its carefully developed instrument performance requirements and associated characterization plan will result in stable and wellunderstood science-quality imagery that will be used for environmental, economic and legal applications. This paper will present a summary of the plan for TIRS-2 prelaunch characterization at the component, subsystem, and instrument level.
\end{abstract}

Index Terms - Landsat 9, TIRS-2, prelaunch characterization, calibration, thermal infrared remote sensing

\section{INTRODUCTION}

Landsat 9 will extend the Landsat data record into its fifth decade once it is launched in December 2020 to the orbit that is now occupied by Landsat 7 and will host two instruments: Thermal Infrared Sensor-2 and Operational Land Imager-2. TIRS-2 and OLI-2 will be near identical builds of TIRS and OLI that are hosted on Landsat 8 which was launched in February 2013. TIRS-2 will produce radiometrically-traceable, geo-located thermal imagery of the Earth with the same parameters as Landsat 8 TIRS: 16day repeat, $100-\mathrm{m}$ spatial sampling, two spectral channels, $185-\mathrm{km}$ swath width, and 70 frames per second operation.

Imagery produced by TIRS-2 will continue the Landsat thermal imagery data record that began for a brief period in 1978 with Landsat 3, and has been nearcontinuous since 1982 with Landsat 4 and 5, and currently Landsat 7 and 8. A few applications that use Landsat thermal imagery are cloud detection and masking, evapotranspiration studies, water use assessments, mapping urban heat fluxes, burnt area mapping, tracking material transport in lakes and coastal regions, and identifying mosquito breeding areas and vector-borne illness potential.

TIRS-2 has a pushbroom design with approximately 15-degree cross-track field of view. The TIRS-2 telescope is nearly telecentric and consists of four elements in two groups of two. The telescope operates at F/1.64 with an effective focal length of $176.7 \mathrm{~mm}$. While the optical prescription of the lenses of TIRS-2 is the same as TIRS, baffles and chamfers have been added to the TIRS2 design to reduce stray light paths found on TIRS shortly after launch of Landsat 8 [1]. The view of TIRS-2 is adjusted between nadir/earth, space, and on-board blackbody with the Scene Select Mechanism (SSM) mirror which rotates around the optical axis of the telescope at a 45-degree angle. The SSM mirror is an ellipse that is diamond-turned aluminum then polished and coated with protected gold. The on-board blackbody (OBB) will nominally be held at $295 \mathrm{~K}$ to provide an on-orbit NISTtraceable radiometric reference. The TIRS- 2 focal plane consists of three quantum well infrared photodector (QWIP) arrays to cover the 15-degree field of view. Each array has $640 \times 512$ detectors with $25-\mu \mathrm{m}$ pitch [2]. Two interference filters are placed over each of the three detectors arrays for spectral selection of the two channels. Each filter spans the full width of the array (640 detector elements) in the crosstrack direction and about the length of 30 detector elements in the along-track direction. Channel 10 is centered at $\sim 10.9$ $\mu \mathrm{m}$ and has $\sim 900$-nm full-width at half maximum response; Channel 11 is centered $\sim 12 \mu \mathrm{m}$ and has $\sim 1000 \mathrm{~nm}$ fullwidth at half maximum.

This paper describes the approach that is being used to characterize the radiometric, spectral, and spatial response of TIRS-2. First, major underlying requirements are discussed followed by description of the experimental set up of calibration light sources. Finally, an overview of the measurement and analysis approach is provided. The paper concludes with status of characterization activities.

Accompanying papers at this conference include Hair et al. [3] covering overall TIRS-2 architecture and 
design, Montanaro et al. [4] detailing testing and analysis of scattered light performance, and Efremova et al. [5] detailing spectral response characterization.

\section{TIRS-2 REQUIREMENTS}

Measurements described in this paper serve to characterize the radiometric, spectral, and geometric response of TIRS-2 and are also used to hold TIRS- 2 performance against various levels of requirements. Table 1 shows some of the main performance requirements and actual performance metrics derived from TIRS are provided for reference.

In addition to these primary performance requirements, there are dozens of Special Calibration Test Requirements (SCTR) that define parameters of characterization measurements such as sampling interval, sampling ranges, and signal-to-noise of the measurement. These requirements are complementary to requirements listed in Table 1 and their associated lower-level requirements since they do not define instrument parameters but rather specify the fidelity and level (component, subsystem, instrument) of the measurement.

Table 1. TIRS-2 requirements alongside TIRS actual performance. Parameters with two values indicate separate values for Channels 10 and 11 , respectively.

\begin{tabular}{lcc}
\hline \hline Requirement & $\begin{array}{c}\text { Landsat } 9 \\
\text { TIRS-2 } \\
\text { requirement }\end{array}$ & $\begin{array}{c}\text { Landsat } 8 \\
\text { TIRS } \\
\text { performance }\end{array}$ \\
\hline \hline NEdT @300K $(\mathrm{K})$ & $<0.4$ & 0.05 \\
NEdL $\left(\mathrm{W} / \mathrm{m}^{2} / \mathrm{sr} / \mu \mathrm{m}\right)$ & $<0.059$, & 0.008 \\
& $<0.049$ & \\
Saturation radiance $\left(\mathrm{W} / \mathrm{m}^{2} / \mathrm{sr} / \mu \mathrm{m}\right)$ & 20.5, & 28.4, \\
& 17.8 & 19.2 \\
40 min 1- $\sigma$ radiometric stability $(\%)$ & $<0.7$ & 0.1 \\
Inoperable detectors $(\%)$ & $<0.1$ & 0 \\
Swath width $(\mathrm{km})$ & $>185$ & 186.2 \\
Ground sample distance $(\mathrm{m})$ & $<120$ & 103.4 \\
Band registration accuracy $(\mathrm{m})$ & $<18$ & 10.4 \\
TIRS-OLI registration accuracy $(\mathrm{m})$ & $<30$ & 20.6 \\
Spatial relative edge slope $\left(\mathrm{m}^{-1}\right)$ & $>0.0047$ & $0.005-0.006$ \\
Spatial edge extent $(\mathrm{m})$ & $<245$ & $200-240$ \\
Absolute radiometric accuracy $(\%)$ & $<2$ & 2,5 \\
Uniformity field-of-view $(\%)$ & $<0.5$ & 1,2 \\
\hline \hline
\end{tabular}

Landsat 8 TIRS did not meet its radiometric accuracy requirements due to a scene-dependent stray light feature that added out-of-field energy to the scene being imaged. Much work was done to develop optical models and algorithms to remove the stray light signal from TIRS imagery in post-processing and results have shown that required radiometric accuracies are achieved [6]. Even though the optical design of TIRS-2 was modified to substantially reduce the stray light paths experienced with TIRS-1, there was an extensive test campaign to demonstrate its improved performance experimentally.

\section{CALIBRATION GROUND SUPPORT EQUIPMENT}

Calibration Ground Support Equipment (CGSE) was developed for TIRS and subsequently used for other projects at NASA Goddard Space Flight Center (GSFC) including TIRS-2. The CGSE is a vacuum-compatible system capable of providing near-ideal and well-understood sources of light to the sensor under test. Most of the primary components of the CGSE are illustrated in Figure 1. The Infrared Source Module (IRSM) consists of a cavity blackbody with two aperture wheels in front that provide various target options. The IRSM is on a $x-y-z$ translation stage that has about 1 -in of range in each direction. Commonly used target apertures consist of $140-\mu \mathrm{m}$ circle, $280-\mu \mathrm{m}$ circle, $2257-\mu \mathrm{m}$ circle, $13470-\mu \mathrm{m}$ square, and $99.8 \times 17403-\mu \mathrm{m}$ slits at different orientations. Next in the optical path is a fold mirror (Fig.1b) followed by an off-axis parabola (OAP, Fig. 1c) that serves as a collimator with approximately $1-\mathrm{m}$ focal length. Finally, the collimated beam shines on a large flat mirror called the steering mirror (Fig. 1d) that rotates in azimuth and elevation about the center of its optical surface. The steering mirror is also on a translation stage that is positioned to allow pointing the beam into the aperture of TIRS- 2 without being vignetted. Fig. 1e is the TIRS-2 telescope and detector subsystem.

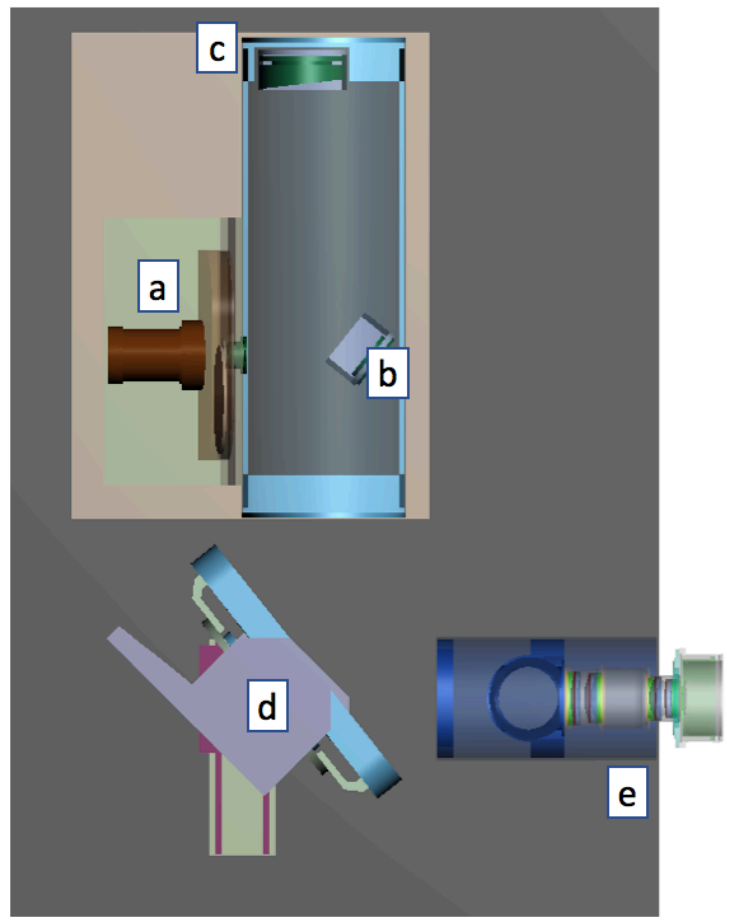

Fig 1. Calibration GSE shown with TIRS-2 telescope and detector subsystem. (a) IRSM, (b) fold mirror, (c) collimator, (d) steering mirror, and (e) TIRS-2 telescope and detector subsystem with baffle simulator.

The CGSE has three primary modes: 1) "Geo-rad" where targets of the IRSM can be pointed to desired locations on the TIRS- 2 image plane for focus, spatial, and some radiometric measurements; 2) spectral mode where the collimator is coupled to a monochromator external to the thermal vacuum chamber rather than the IRSM; and 3) a 
large extended blackbody source that is a v-grooved plate called the 'flood source.' Figure 1 illustrates the "geo-rad mode and Figure 2 illustrates the spectral and Flood source modes of the CGSE with the full TIRS-2 instrument.

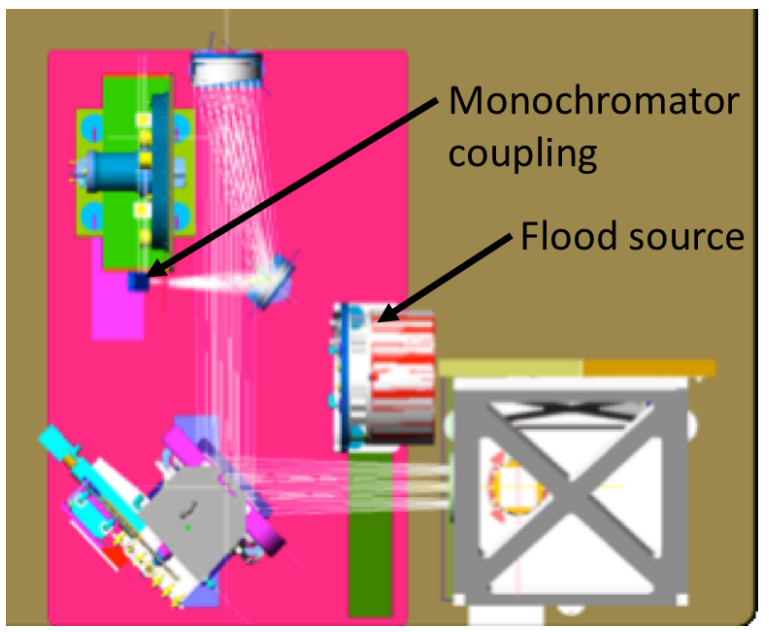

Fig 2. Calibration GSE shown with TIRS-2 instrument-level system for a), and b) spectral mode where a monochromator outside of the thermal vacuum chamber is coupled to the collimator. Subset (b) also indicates the flood source which is moved on a linear stage (green rectangle) to be in front of TIRS-2 for radiometric testing.

\section{TIRS-2 CHARACTERIZATION}

TIRS-2 telescope and detector subsystem testing began in November 2017 and continued through early March 2018 and is called the TIRS Imaging Performance and Cyroshell evaluation (TIPCE). The primary goal of TIPCE was 1) to provide focus information so that optical and mechanical teams can fabricate shims that optimize the alignment of the image plane with the detector; 2) perform wide-field stray light survey measurements to assess performance of the new baffle design, and 3) provide initial assessment of spatial and spectral performance though not with the full optical path of TIRS-2. Scattered light characterization is emphasized at this moment in the TIRS-2 build since it is the earliest that baffle performance can be demonstrated on the flight system and the smaller size of the telescopedetector subsystem allows closer proximity to the CGSE than the full instrument and therefore it can be illuminated at much greater field angles. Results of scattered light measurements are presented in Montanaro et al 2018 [4] in these proceedings.

There were three TIPCE thermal vacuum campaigns: TIPCE-1 during Nov 16-19, 2017; TIPCE-2 during Jan 1-Feb 3, 2018; and TIPCE-3 during Feb 27-Mar 3, 2018. Detector electrical current parameters became a concern after TIPCE-1 and the project decided to switch to the spare detector set. This means that TIPCE-2 was starting with telescope-detector alignment based on mechanical knowledge of the system rather than TIPCE-1 test results and explains the extended period between TIPCE- 1 and -2 .
The spatial response of TIRS-2 is the twodimensional weighted function of the spatial sensitivity a single detector element has on the surface of the earth. Two spatial performance metrics are illustrated in Figure 3 and are used to simplify requirements definition and analysis: relative slope of the edge in the $40-60 \%$ range and the spatial extent of that edge in the $10-90 \%$ of the normalized signal.

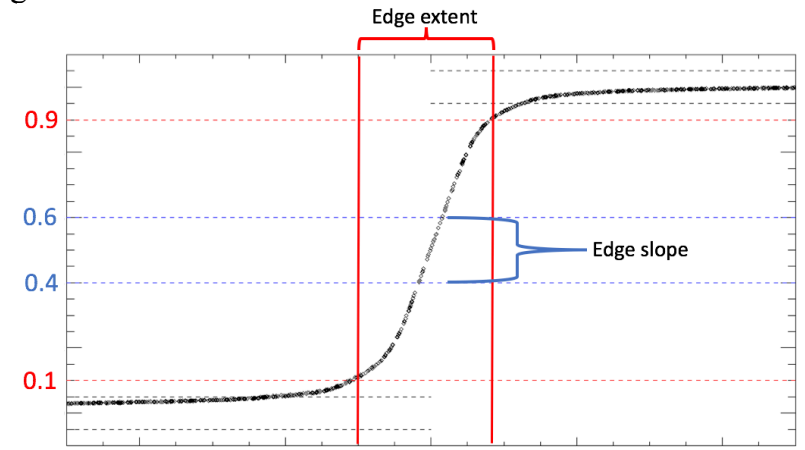

Fig. 3. Measurements with the $2257-\mu \mathrm{m}$ CGSE target are used to generate an edge response. The edge extent and edge slope spatial performance metrics are derived from this function.

In the final subsystem test, TIPCE-3, measurements were taken to characterize these metrics at five locations per channel per detector array which totals 30 locations. Figures 4 and 5 show these results for the 10.9$\mu \mathrm{m}$ channel as blue diamonds across the combined 1920detector wide array. Results from other TIPCE campaigns are also plotted to show consistency and the red dashed line indicates the required performance. Note that a different detector was integrated for TIPCE-1. All spatial results for both channels meet edge slope and edge extent requirements including variability, ripple metrics, and sampling metrics across the TIRS-2 15-degree field of view.

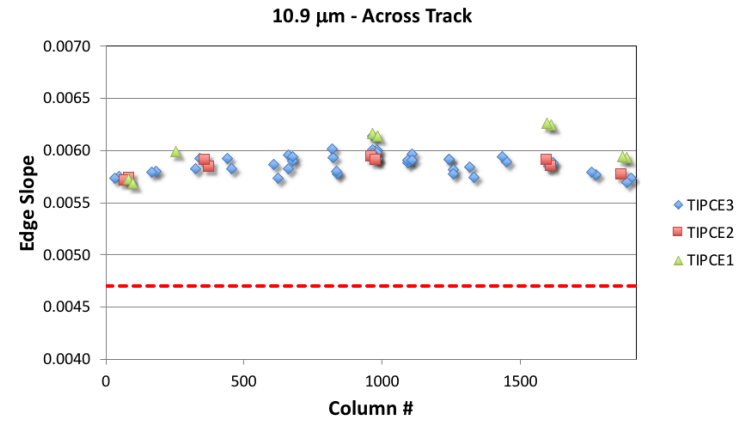

Fig. 4. Spatial edge slope results for the 10.9- $\mu \mathrm{m}$ channel from the three subsystem-level tests. 


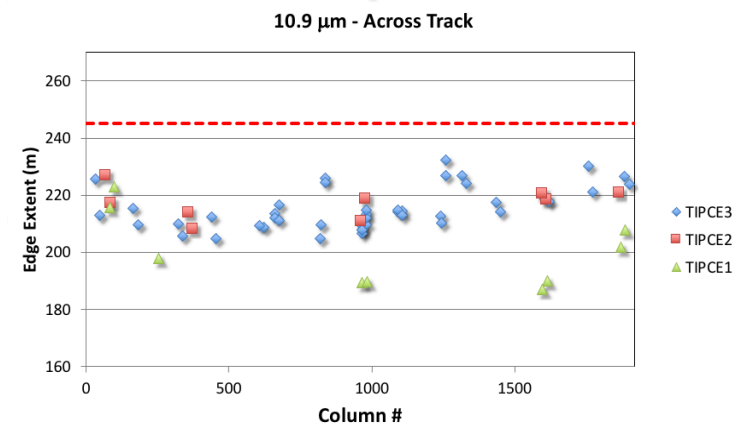

Fig. 5. Spatial edge extent results for the 10.9- $\mu \mathrm{m}$ channel for the three subsystem-level tests.

The spectral response of TIRS-2 is derived using two methods: component-level stack up and system-level measurements. It is difficult to test the filter and detector components under the conditions they will operate at the system level, namely the F/\# of the beam used for characterization. Therefore, the system-level measurements will be used for the operational system while the component level results serve as reliable confirmation. Detailed TIRS-2 spectral results are discussed in Efremova et al 2018 [5], but an example of the relative spectral response (RSR) is shown in Figure 6 along with an example of performance against one of the several spectral requirements is shown in Figure 7.

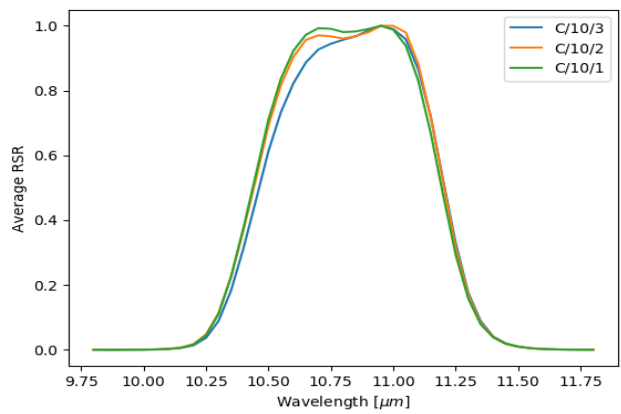

Fig 6. TIRS-2 10.9- $\mu \mathrm{m}$ channel RSR derived from TIPCE-3 measurements. The three lines show the response for three locations on one of the three detector arrays.

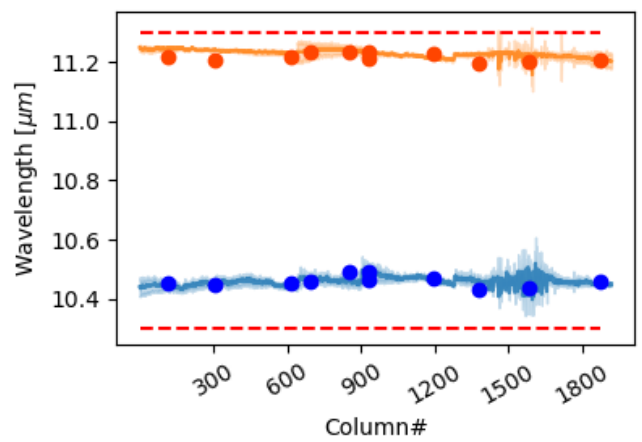

Fig 7. $10.9-\mu \mathrm{m}$ channel spectral band edge requirement is indicated with the red dashed lines, TIPCE-3 results are shown with solid circles (orange for the upper band edge and blue for the lower band edge), and component-level predictions are shown with the solid line.
The radiometric response of TIRS-2 will be traceable to NIST via the flood source which received NIST-traceable characterization January 2018 and will undergo a repeat characterization after TIRS-2 instrumentlevel testing. The flood source has a $0.3 \%(\mathrm{k}=1)$ uncertainty while the propagation to TIRS-2 is estimated to increase this value to $0.6 \%$. Combined with other error sources, including scattered light, the total radiometric uncertainty of TIRS-2 is expected to be $1.7 \%$ or better.

\section{CONCLUSION}

The TIRS-2 Calibration team formulated comprehensive characterization plan for component-, subsystem-, and instrument-level testing. These plans were reviewed by internal and external experts in the form of two Engineering Peer Reviews that were held before the overall Preliminary (June 2016) and Critical (Feb 2017) Design Reviews. TIRS2 telescope and detector subsystem-level testing successfully validated stray light mitigation and provided early assessment of spatial and spectral performance that indicates instrument-level requirements will be met. The Calibration team is currently finalizing details of instrument-level testing planned for Fall 2018 - Spring 2019. The Calibration team will continue to support TIRS-2 through delivery in August 2019, subsequent integration to the spacecraft, and post-launch checkout after the expected Landsat 9 launch in December 2020.

\section{REFERENCES}

[1] M. Montanaro, A. Gerace, A. Lunsford, D. Reuter, "Stray Light Artifacts in Imagery from the Landsat 8 Thermal Infrared Sensor," Remote Sensing, vol. 11, pp. 10435-10456, 2014.

[2] M. Jhabvala, D. Reuter, K. Choi, C. Jhabvala, M. Sundaram, "QWIP-based Thermal Infrared Sensor for the Landsat data continuity mission,” Infrared Phys. Technol. 2009, 52, 424-429.

[3] J.H. Hair, M. Djam, S.L. Tonn, D.C. Reuter, A.A. Simon, J. McCorkel, M. Montanaro, "Landsat 9 Thermal Infrared Sensor 2 Architecture and Design Overview," These proceedings.

[4] M. Montanaro, J. McCorkel, J. Tveekrem, J. Stauder, J.H. Hair, D.C. Reuter, "TIRS-2 preliminary stray light assessment," These proceedings.

[5] B. Efremova, A.J. Pearlman, J. McCorkel, M. Montanaro, M. Hickey, A. Lunsford, D.C. Reuter, "Landsat 9 Thermal Infrared Sensor 2 Preliminary Spectral Test Results," These proceedings.

[6] A. Gerace, M. Montanaro, "Derivation and validation of the stray light correction algorithm for the thermal infrared sensor onboard Landsat 8," Remote Sensing of Environment, vol. 191, pp. 246-257, 2017. 